\title{
Repeatability and randomness in heterogeneous freezing nucleation
}

\author{
G. Vali \\ Department of Atmospheric Science, University of Wyoming, Laramie, WY 82071, USA
}

Received: 3 January 2008 - Published in Atmos. Chem. Phys. Discuss.: 27 February 2008

Revised: 24 July 2008 - Accepted: 4 August 2008 - Published: 29 August 2008

\begin{abstract}
This study is aimed at clarifying the relative importance of the specific character of the nuclei and of the duration of supercooling in heterogeneous freezing nucleation by immersed impurities. Laboratory experiments were carried out in which sets of water drops underwent multiple cycles of freezing and melting. The drops contained suspended particles of mixtures of materials; the resulting freezing temperatures ranged from $-6^{\circ} \mathrm{C}$ to $-24^{\circ} \mathrm{C}$. Rank correlation coefficients between observed freezing temperatures of the drops in successive runs were $>0.9$ with very high statistical significance, and thus provide strong support for the modified singular model of heterogeneous immersion freezing nucleation. For given drops, changes in freezing temperatures between cycles were relatively small $\left(<1^{\circ} \mathrm{C}\right)$ for the majority of the events. These frequent small fluctuations in freezing temperatures are interpreted as reflections of the random nature of embryo growth and are associated with a nucleation rate that is a function of a temperature difference from the characteristic temperatures of nuclei. About a sixth of the changes were larger, up to $\pm 5^{\circ} \mathrm{C}$, and exhibited some systematic patterns. These are thought to arise from alterations of the nuclei, some being permanent and some transitory. The results are used to suggest ways of describing ice initiation in cloud models that account for both the temperature and the time dependence of freezing nucleation.
\end{abstract}

\section{Introduction}

While it is widely recognized that ice nucleation in tropospheric clouds is of critical concern for weather and climate models, there are major shortcomings in our ability to treat this process reliably. Lack of adequate measurement capa-

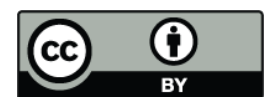

Correspondence to: G. Vali (vali@uwyo.edu) bilities, enormous variability of conditions and weaknesses in theory all contribute to this situation. These issues lead to rather different and uncertain assumptions in cloud models about how to represent heterogeneous freezing (e.g. Kärcher and Lohmann, 2003; Khvorostyanov and Curry, 2004; Diehl and Wurzler, 2004; Morrison et al., 2005; Liu and Penner, 2005; Prenni et al., 2007).

One of the areas of uncertainty in the formulation of heterogeneous ice nucleation is the separation between temperature dependence and time dependence. This paper attempts to clarify that issue for immersion freezing. Based on laboratory experiments, the relative roles of temperature and timedependence are assessed. It is shown that the former has primary importance, and the possible contribution of the second is quantified.

\section{Underlying concepts}

The fundamental view of nucleation of a stable phase from the metastable parent phase is that the nucleation event is the result of fluctuating growth of an embryo. Fluctuations in size are, principally, molecule by molecule and growth becomes favored over diminution once the embryo reaches a critical size that is defined by macroscopic conditions temperature in the case of freezing. At a given temperature, fluctuations lead to a certain probability that a critical embryo develops. In the classical nucleation theory (CNT), as formulated by Volmer (1939), this is usually expressed as a nucleation rate, $J$, which is the number of embryos reaching critical size, on average, per unit volume and per unit time. An expression for the nucleation rate as a function of temperature, with appropriate values for the ice/water interfacial energy and other constants, is adequate to describe homogeneous freezing nucleation; observations and theory converge to indicate values of $J=10^{0} \ldots 10^{12} \mathrm{~cm}^{-3} \mathrm{~s}^{-1}$ in the range $-33 \ldots-40^{\circ} \mathrm{C}$. For a given volume, the nucleation rate

Published by Copernicus Publications on behalf of the European Geosciences Union. 
is a direct predictor of the probability of nucleation within unit time interval.

In heterogeneous freezing nucleation, a substrate immersed in the supercooled liquid facilitates the phase transition by providing the possibility for an ice embryo to form on it and reach critical size ${ }^{1}$ with a lower free energy barrier than would be the case for an isolated embryo (homogeneous nucleation). The nature of the substrate determines the degree of energy reduction and hence the temperature at which freezing becomes likely on the scale perceivable in an experiment. For a uniform surface, the nucleation rate can be derived using the modified energy barrier and given as the number of embryos of critical size forming per unit time and per unit surface area of the substrate (e.g. Fletcher, 1970; Pruppacher and Klett, 1997). There is no conceptual difficulty with this and the results of the theory are, in principle, testable. Even if the substrate surface contains preferred sites for embryo growth, such as steps or dislocations, but those are all considered equal and randomly distributed, a simple modification to the formulation is possible by incorporating a probability of the preferred sites ${ }^{2}$ per unit area.

Difficulties arise when the sites are not equal in their effectiveness, and if, additionally, the substrate material is a dispersion of various particle sizes and/or composition. Since the characteristics of surface features that may constitute the sites are not known, and thus no a priori descriptions can be made, the effectiveness of sites has to be measured and that is where varying assumptions about the relative roles of site characteristics and of time become important and when the definition of nucleation rate $J$ can become ambiguous. Similarly, even if assumptions were to be made about the sites, the predicted behavior would still need to take into account the role of time.

\section{Stochastic and singular descriptions}

The fundamentally opposing views of stochastic versus singular behavior of freezing nucleation can be traced in most explicit fashion to Bigg (1953), Carte (1959), Dufour and Defay (1963) for the stochastic model, and to Dorsey (1938), Levin (1950) and Langham and Mason (1958) for the singular model. The terminology characterizing the two assumptions in this way was introduced by Vali and Stansbury (1966), following similar definitions by Dorsey (1948). In essence, the stochastic model assigns a probability per unit time for nucleation to take place in a given volume of water, with the magnitude of the probability depending on the im-

\footnotetext{
${ }^{1}$ Critical size is used here to describe the embryo dimension beyond which the probability of growth exceeds that of diminution. This is a useful shorthand designation since the stability of an embryo will depend on more than one parameter, as determined by the specific form or shape of the particular embryo.

${ }^{2}$ These are often referred to as "active sites", but here will be just called "sites".
}

purities it is expected to contain as a simple proportion to the average properties of the sample, so that all equal volumes, drops, have the same chance to freeze within a period of time. In contrast, the singular model considers that each impurity or site has a characteristic temperature, $T_{c}$, at which it causes nucleation as the sample is cooled. Chance allocation of nuclei into drops is expected to result in each drop having a different set of $T_{c}$ values in the temperature range where the average number per drop is small. In its basic form, the singular description would lead to instantaneous freezing at $T_{c}$, and further time at that same temperature would lead to no additional nucleation events in a set of drops.

A few examples can illustrate how experiments can provide support for the stochastic description. Vonnegut and Baldwin (1984) observed large variations in the lapse of time (from a few seconds to more than $5 \mathrm{~min}$ ) that was needed for nucleation to occur when a single sample of water containing silver iodide particles was repeatedly cooled to the same temperature. By analogy with radioactive decay, they interpreted these observations as a confirmation of the stochastic model. More recently, Seeley and Seidler (2001a, b) and Zobrist et al. (2007) reported on experiments in which drops of water coated with nonadecanol and other aliphatic alcohols were exposed to repeated cycles of cooling until nucleation was observed, followed by melting. By equating the number of times that a drop was observed to freeze within a temperature interval with the fraction of a large number of drops expected to freeze in the same interval when cooled simultaneously, the conclusion is reached that the observed changes in nucleation temperatures for any given drop could be accounted for on the basis of a nucleation rate derived from classical nucleation theory (CNT) with appropriately fitted parameters, i.e. that the variations in freezing temperatures between trials resulted from stochastic effects.

The most fundamental evidence in support for the singular description comes from numerous sets of observations (e.g. Dorsey, 1938) that when a sample of water is subdivided into smaller parts, each of the resulting volumes freezes at different and more or less repeatable temperatures. What has proven to be difficult is to show that whatever differences appear are not the result of random fluctuations. One approach to do that is to examine the dependence of the average freezing temperature on sample volume. However, it can be shown (the Appendix in Vali and Stansbury, 1966; page 351 in Pruppacher and Klett, 1997) that both descriptions lead to the same result if the probability density function (pdf) of $T_{c}$ follows an exponential form. Since the exponential is a good approximation in many cases, volume-dependence turned out to be a weak test. A basic objection to the singular description is that it disregards the consequences of fluctuating embryo growth.

Based on experiments in which arrays of drops were subjected to steady cooling and interjected periods at constant temperatures, Vali and Stansbury (1966) suggested a qualitative description that allowed for stochastic effects to 
modify freezing temperatures corresponding to the characteristic temperatures of the suspended impurities. The effects of random variations was indicated to be equivalent to about $1{ }^{\circ} \mathrm{C}$ variations in characteristic temperatures. That "modified singular description" was given further support by experiments described in Vali (1994). Quite recently, Marcolli et al. (2007) reported on experiments in which drops containing mineral dust were subjected to repeated freezing cycles and concluded that a nucleation rate function did not account for the observations, and that it was necessary to include in the description different nucleating abilities for particles in different drops. This work, therefore, supports the modified singular model.

The experiments to be reported here were undertaken to provide a quantitative basis for assessing the stochastic contribution to freezing probabilities for samples that contain impurities with large ranges of characteristic temperatures.

\section{Experimental and analysis procedures}

The experiments consisted of repeated freezing and melting of drops on a cold stage. Typically, 100 to 144 drops of $0.01 \mathrm{~cm}^{3}$ volume were used in an experiment and up to 65 cycles of freezing and melting were executed. Drops were placed on a hydrophobic surface (thin plastic sheet or aluminum foil coated with a silicone varnish) on top of a $10 \times 10 \mathrm{~cm}$ and $1-\mathrm{cm}$ thick copper cold stage cooled by thermoelectric modules ${ }^{3}$. All drops were dispensed from the same bulk sample, i.e. the distribution of particles among the drops was a chance process. The cold stage temperature was lowered at a constant rate of $1^{\circ} \mathrm{C} \mathrm{min}-1$ until all drops were frozen, after which it was raised to about $+5^{\circ} \mathrm{C}$ for $5 \mathrm{~min}$. The total time at positive temperatures was considerably longer than the time necessary to melt the drops. Freezing of the drops was detected by the change from clear to opaque appearance and was evaluated from time lapse photographs. Temperatures were recorded with a resolution of $0.27^{\circ} \mathrm{C}(10 \mu \mathrm{V}$ output from the thermocouple sensor). The precision of the recorded nucleation temperatures was limited by the sensitivity of the visual detection of brightness changes, and because of that the recorded temperatures are colder than the actual initiation of freezing by an estimated $0.2 \ldots 0.5^{\circ} \mathrm{C}$.

The influence of the surface supporting the drops is of obvious concern in this type of experiment. In this regard, the first control is that the lowest temperatures at which drops of distilled water were observed to freeze was near $-25^{\circ} \mathrm{C}$; that limit may have been set by the surface or by the degree of purity of the distilled water used. The influence of the surface was further tested by observing the freezing temperatures of drops, melting them, and then freezing them again after shifting them to a new position. The shift could be accomplished

\footnotetext{
${ }^{3} \mathrm{~A}$ description of the apparatus is given in Vali and Stansbury (1966).
}

by gently pushing the drops with a fine steel rod (syringe needle); generally the amount of shift was about two or three times of the diameter of drop. These tests showed that the drops retained their freezing temperatures to the same degree as when they were left in the same position. On the average the changes were $<0.1^{\circ} \mathrm{C}$ although the range of changes extended to about $\pm 2^{\circ} \mathrm{C}$; this can be compared with the results to be presented later. Many such tests were conducted with different samples and over long periods of time. The conclusion seems warranted that the supporting surface did not influence the measurements.

Because repeatability was one of the quantities to be evaluated from the experiments, the possibility of neighboring drops influencing one another was of special concern. Frost spreading on the supporting surface was observed in runs with poorly prepared coatings of the surface and with high humidity in the air. For this reason, the enclosure covering the drops included a cold trap to dry the air. This resulted in slow evaporation of the drops, but experiments extending to over $30 \mathrm{~h}$ were still possible. The absence of neighbor effects was tested in special runs in which a checkerboard pattern was created with two kinds of drops: some containing particles that causes early freezing and distilled water drops. The distilled water drops remained unfrozen even with all the interspersed drops frozen. Furthermore, data from the main experiments was used to evaluate any change in the likelihood of freezing of a drop due to an earlier freezing event next to it; no such effect was found. In all, there is fair assurance that the data are free of artifacts.

The distilled water used in the experiments was obtained from a glass still of average quality. The emphasis in the use of this water was on reproducibility, not on high purity. To aid this, a large quantity was stored in a container of $20 \mathrm{~L}$ capacity and smaller amounts were withdrawn from the container for the experiments. Suspensions of soils samples were obtained by adding about a gram of soil to $250 \mathrm{~mL}$ of distilled water and the coarse matter was allowed to settle over a day or so. The supernatant was further diluted as needed, with the main criterion being to produce a large range of freezing temperatures for the drops to be tested; the importance of that feature will be discussed later. Drops were produced with a disposable syringe and stainless steel needle. The uniformity of drop volumes depended on the skill of the operator; while it could be judged by eye quite sensitively it has not been quantitatively evaluated.

Differential and cumulative nucleus spectra, $k(T)$ and $K(T)$, were calculated following Vali (1971):

$$
\begin{aligned}
& k(T)=\frac{1}{V} \frac{1}{N(T)} \frac{d N}{d T} \\
& K(T)=\frac{\ln N_{o}-\ln N(T)}{V}
\end{aligned}
$$


where $N_{0}$ is the total number of drops in the sample and $N(T)$ is the number not frozen at $T$. The dimension of $k(T)$ is $\left[\mathrm{cm}^{-3} \mathrm{deg}^{-1}\right]$ for $V$ in $\left[\mathrm{cm}^{3}\right]$, and that of $K(T)$ is $\left[\mathrm{cm}^{-3}\right]$. Differences in freezing temperatures between two successive runs for a given drop, $\delta T_{i j}$, were calculated as the later versus the earlier temperature, so that negative values indicate a decrease with time, and positive ones an increase with time. The subscript $i$ is the earlier run number and $j$ is the drops number. Rank correlation coefficients for pairs of runs were calculated for drops ordered according to decreasing freezing temperatures.

Analyses were done at two levels. First, for entire sequences of runs and including all the drops. Second, for drops most likely to contain only one nucleus active above a chosen threshold temperature, and, in some cases, for a subset of the runs that showed the most uniform trends. The "single-nucleus" (SN) subsets was defined so as to restrict analyses to those drops for which changes in freezing temperatures were not likely to be due to one or another nucleus becoming active in successive tests. For samples like the ones used here, having a large spread of freezing temperatures for a given set of drops, restricting analyses to an SN subset is somewhat unnecessary, however for samples that have narrow ranges of freezing temperatures the distinction would be important. The SN subset was defined as follows: It is assumed that the apportioning of nuclei of given kinds among the drops follows a Poisson distribution. For a drop frozen at some temperature $T$ there was at least one nucleus in that drop active in the interval $0^{\circ} \mathrm{C} \ldots T$. The average number of nuclei per drop with freezing temperatures above $T$ is $n(T)=K(T) \times V$ where $V$ is the volume of one drop. Then, the probability for a drop of volume $V$ to contain two or more nuclei of the same kind is given by:

$P=1-e^{-n(T)}-n(T) e^{-n(T)}$

This expression contains the probabilities of zero and one event, for an average event frequency of $n(T)$, subtracted from unity. By comparing Eqs. (2) and (3) it can be seen that $P$ is directly related to $N / N_{0}$. For the analyses given here, Eq. (3) was used to find the temperature $T_{s n}$ corresponding to $P=0.1$, which means that $N\left(T_{s n}\right) / N_{0}=0.58$. Drops frozen at $T_{s n}$ in the first of a group of runs constitute the SN subset. The choice of P-value used to make this separation is a compromise between better assurance and overly small sample size. It should be noted that there is no full assurance that the goal of the SN subset is fulfilled. First, because it is based on a statistical estimate. Second, since the selection of a subset was based on the freezing temperatures observed in the first run of a sequence, loss of the specific nucleus responsible for freezing in that run could result later in freezing at lower temperatures where the probability of multiple similar nuclei was high. However, since most changes were small in comparison to the range of temperatures over which the drops in a sample froze, deviations from the SN subset definition due to this fact, while undoubtedly present to some degree, can be considered to be of minor importance. Fuller justification of this statement will emerge from the results to be presented.

\section{Results}

\subsection{Soil sample A}

Surface soil from the near Red Deer, Alberta, Canada was used for two sets of experiments a few months apart. The sample contained some vegetative material, both recent and decayed, and was passed through a coarse sieve before use to remove large pieces. In addition, the sample was allowed to settle after mixing the powder into distilled water and only the supernatant was used. The supernatant was optically clear to the eye, but even so, the concentration of particles is estimated to have exceeded $10^{8} \mathrm{~cm}^{-3}$. No effort was made to identify either the chemical composition or detailed physical state of the nucleating agents. Clearly, with complex samples like this, it is not feasible even in principle to determine the size distribution of the particles that carry the nuclei. Those particles represent a minute fraction of the total amount of particulate in the sample. In spite of the lack of definiteness about the nature of the nucleating material, this type of sample preparation was chosen because it was known (Schnell and Vali, 1972) to result in suspensions that are stable over periods of days and that contain nuclei active at temperatures as high as $-6^{\circ} \mathrm{C}$.

Sample A refers to the most extensive of the several sets of tests conducted. In this set of runs, 96 drops were used along with 48 drops of distilled water for control. A total of 55 runs were made, the first 50 without interruption and the last 5 after an overnight gap. Six of these runs were omitted from the analysis because they had more than one missing data point. Each cycle was continued until all drops were frozen, including the distilled water drops. The average duration of a run during the first day, including the time to melt the drops, was $17 \mathrm{~min}$. At the final dilution used, the range of freezing temperatures in the initial run was -6.4 to $-18.6^{\circ} \mathrm{C}$. The mean freezing temperature of the 96 drops was $-13.8^{\circ} \mathrm{C}$ in the first run and changed during the sequence as shown in Fig. 1a. Figure $1 \mathrm{~b}$ shows the numbers of drops that froze per half-degree intervals in the first and last runs. Figure 1c shows the cumulative spectra (Eq. 2) for the first and last runs. It is worth noting that the overnight gap after run \#50 didn't produce changes much different from the rest of the sequence.

Run-to-run changes in freezing temperatures, $\delta T_{i j}$, over all values of run number $i$ and drop number $j$ of the full set of runs are characterized in Row 1 of Table 1 as temperature values for indicated percentiles of the overall frequency distribution. The median of the 4068 values of $\delta T_{i j}$ is zero, and the distribution is symmetrical except at the extremes. Although a small number of $\delta T_{i j}$-values extend to $\pm 4^{\circ} \mathrm{C}$, $50 \%$ of the changes are within $\pm 0.27^{\circ} \mathrm{C}$ (the resolution of 
Table 1. Distribution of run-to-run temperature changes, $\delta T_{i j}\left({ }^{\circ} \mathrm{C}\right)$ for soil sample A.

\begin{tabular}{|c|c|c|c|c|c|c|c|c|c|c|c|c|c|c|}
\hline \multirow[t]{2}{*}{ Row } & \multirow[t]{2}{*}{ Data } & \multicolumn{13}{|c|}{ Percentile } \\
\hline & & 0.1 & 0.5 & 1 & 5 & 10 & 25 & 50 & 75 & 90 & 95 & 99 & 99.5 & 99.9 \\
\hline 1 & Runs \#1...55 (4068 values) & -4.4 & -3.2 & -2.4 & -1.1 & -0.53 & -0.27 & 0 & 0.27 & 0.53 & 1.1 & 2.4 & 3.2 & 5.1 \\
\hline 2 & SN subset (1840 values) & -3.9 & -3.1 & -2.2 & -1.1 & -0.53 & -0.27 & 0 & 0.27 & 0.53 & 0.8 & 2.1 & 2.9 & 5.1 \\
\hline 3 & Random order (see Sect. 6) & -10.4 & -9.9 & -9.6 & -8.3 & -6.9 & -2.7 & 0 & 2.9 & 6.9 & 8.5 & 9.5 & 9.9 & 10.4 \\
\hline
\end{tabular}

(a)

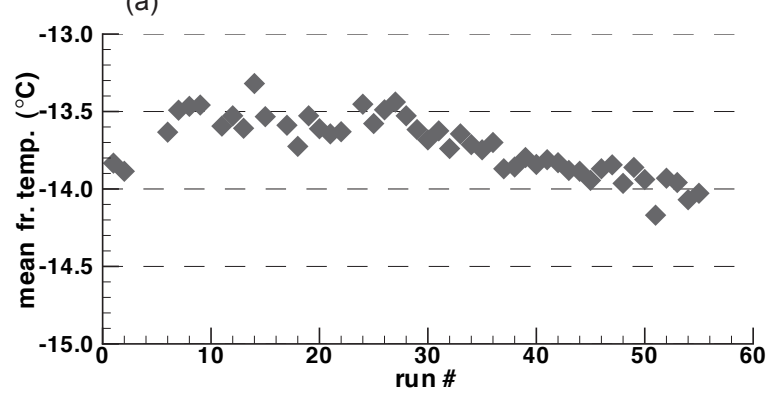

(b)

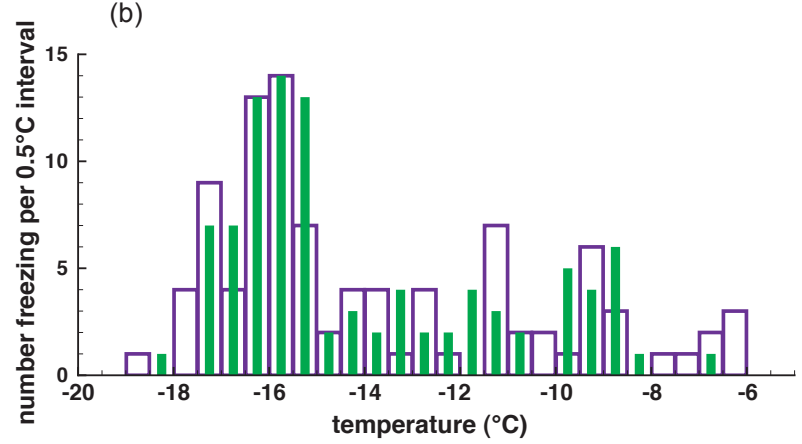

(c)

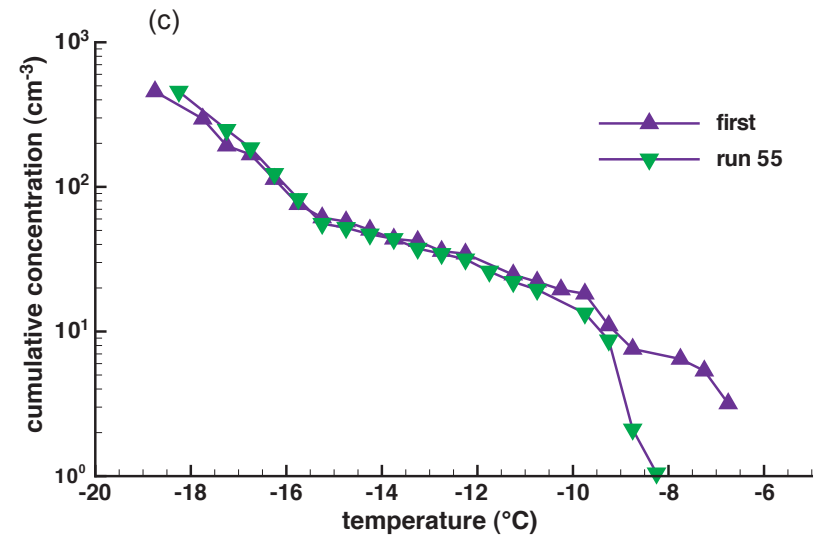

Fig. 1. (a) Average freezing temperatures for 96 drops of Sample A in a sequence of 55 runs. (b) Histogram of the numbers of drops of freezing as a function of temperature in the first and last runs of the sequence. (c) Cumulative spectra, expressing the volume concentration of nuclei which caused freezing above the indicated temperatures. (a)

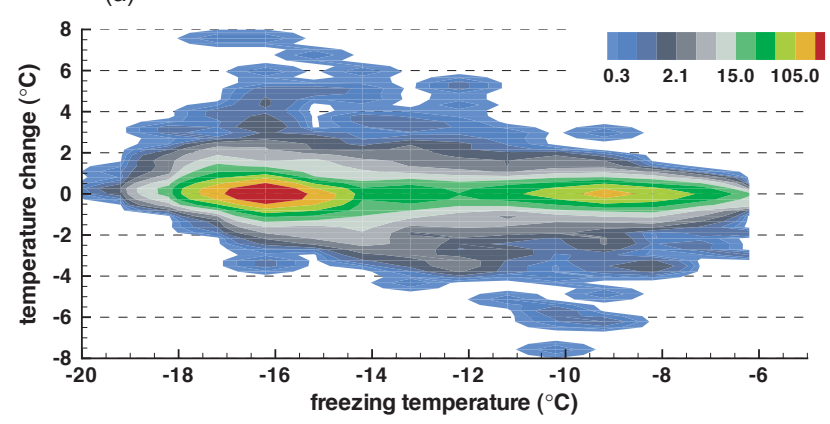

(b)

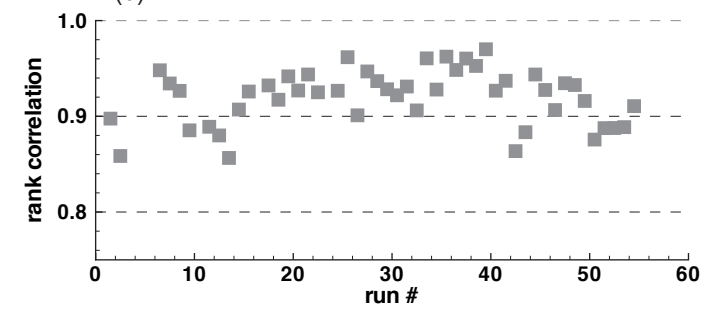

Fig. 2. (a) Contour plot of the number of observed changes in temperatures from one run to the next for drops freezing at temperatures indicated on the abscissa, for soil sample A. The colored density scale is logarithmic and is in units of $0.27\left({ }^{\circ} \mathrm{C}\right)^{2}$. (b) Rank correlation coefficients of the freezing temperatures in successive runs.

the temperature readings). Figure 2a shows a contoured frequency plot of $\delta T_{i j}$-values as a function of the freezing temperature in the earlier run. The clustering of high frequencies around $-9^{\circ} \mathrm{C}$ and $-16^{\circ} \mathrm{C}$ is a reflection of distribution of freezing events shown in Fig. 1b. This contour plot demonstrates that the majority of changes fell in a narrow, symmetrical band about zero, independent of the actual temperature.

The fact that the great majority of $\delta T_{i j}$, values are very small compared to the spread of freezing temperatures for the population of drops provides strong evidence against randomness. But, the repeatability of freezing temperatures is most clearly demonstrated by Fig. $2 b$ showing the Spearman rank correlation coefficients ${ }^{4}$ (IDL software from Research Systems Inc.) for freezing temperatures observed in subsequent pairs of runs. The rank correlation is independent of

\footnotetext{
${ }^{4}$ Correlation of the order in which drops are observed to freeze in one run versus the order of freezing in the other run.
} 
(a)

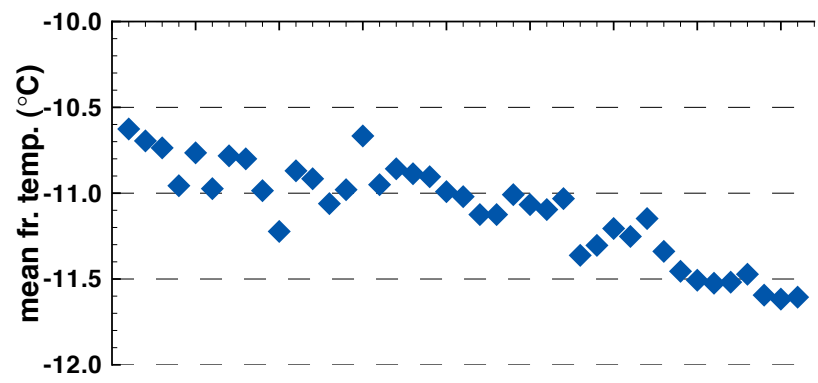

(b)
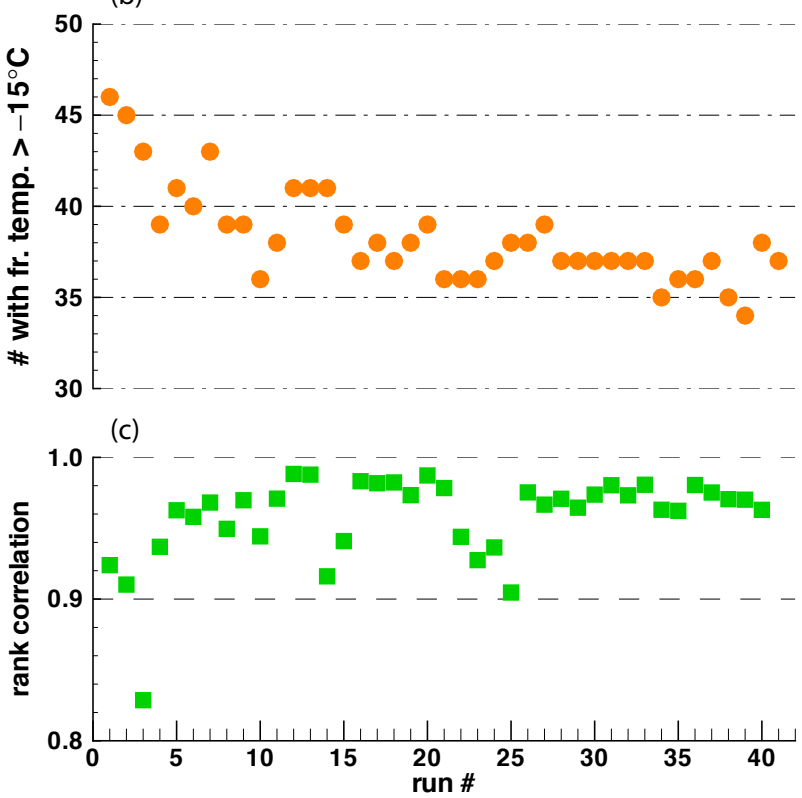

Fig. 3. Data for the SN subset (see text for definition) of soil sample A. (a) Average freezing temperatures. (b) Number of drops freezing above the selection threshold of $-15^{\circ} \mathrm{C}$. (c) Rank correlation coefficients of the freezing temperatures in successive runs.

changes in actual temperatures and focuses on the relative order in which drops freeze in a run; in a sense, this is a more severe test than the more conventional correlation in cases where changes in absolute values are involved. For the sequence in Fig. 2b, the average value of the rank correlation coefficients is 0.92 and the standard deviation of the 54 values is 0.29 . The two-sided significance that these rank correlations arose by chance is $3 \times 10^{-30}$. The mean value of the linear (Pearson) correlation coefficients of temperatures for subsequent pairs of runs is 0.96 and the standard deviation of the 54 values is 0.043 . It is clear, that both types of correlations provide strong support for the repeatability of freezing temperatures.

The repeatability of the freezing temperatures can be further demonstrated by comparing the correlation coefficients for the freezing temperatures actually observed with what would result if the temperature values were randomly mixed, thereby simulating a stochastic source as determinant of the temperature at which any drop freezes. The coefficient of correlation between the freezing temperatures in runs \#26 and \#27 is 0.98 . When the freezing temperatures of run \#27 are randomly re-ordered 100 times, the range of values of the correlation coefficient is found to be -0.25 to +0.31 (1and 99-percentiles). Thus, if each drop in run \#27 had an equal probability to freeze, within the pdf of observed freezing temperatures, the correlation with run \#26 would have fallen in that range. That makes the observed value of 0.98 clearly incompatible with random freezing.

The SN subset (as defined in Sect. 4) was constructed using runs \#6 to \#50 and drops with $T>-15.2^{\circ} \mathrm{C}$ in run \#6. The SN subset consists of 46 drops in 41 runs. Its main characteristics are shown in Fig. 3: panels (a) and (c) are equivalent to Figs. 1a and $2 b$ while Fig. $3 b$ shows how many of the 46 drops retained freezing temperatures $>-15.2^{\circ} \mathrm{C}$ during the sequence. As Row 2 in Table 1 shows, the majority of the run-to-run changes are not significantly different from those for the full data set but there are fewer extreme values. Looking at the magnitudes of fluctuations drop by drop, it is found that the mean values over the 41 runs fall between $-0.11^{\circ} \mathrm{C}$ and $+0.047^{\circ} \mathrm{C}$ for $90 \%$ of the 46 drops. The largest negative value is $-0.2^{\circ} \mathrm{C}$ and the largest positive value is $+0.07^{\circ} \mathrm{C}$. The mean and standard deviation of the 40 values of the rank correlation coefficient are 0.96 and 0.03. By coincidence, the same values are obtained for the linear correlation coefficients.

In addition to the statistical measures summarized above, the nature of the changes deserve a closer look. Figure 4 shows the freezing temperatures of 11 (every fourth one) of the drops in the SN subset. Several of these drops froze at essentially the same temperature throughout the sequence, others exhibit sudden large shifts, some underwent gradual changes at least in part of the sequence, and some changes appear temporary. These patterns are typical, although with many variations, of what has been seen in these experiments. A more complete set of drop histories is included in the Supplement.

\subsection{Soil sample B}

This sample was prepared with the same soil as sample A and handled in a similar way. There was no assurance that the small amount of soil withdrawn for suspension would be identical in composition or amount, neither was control for the length of time that the sample was in suspension before the experiments were performed. These variations account for the slight differences in the results.

Repeated freezing of 110 drops was carried out over 3 days. Twelve runs on the first day, 30 on the second, and 12 on the third. Eleven drops of distilled water were also included for control, but in this sequence, differently from sample A, cooling was discontinued once all of the drops with soil suspension were frozen. Only one drop of distilled water was observed to freeze during the whole sequence of 

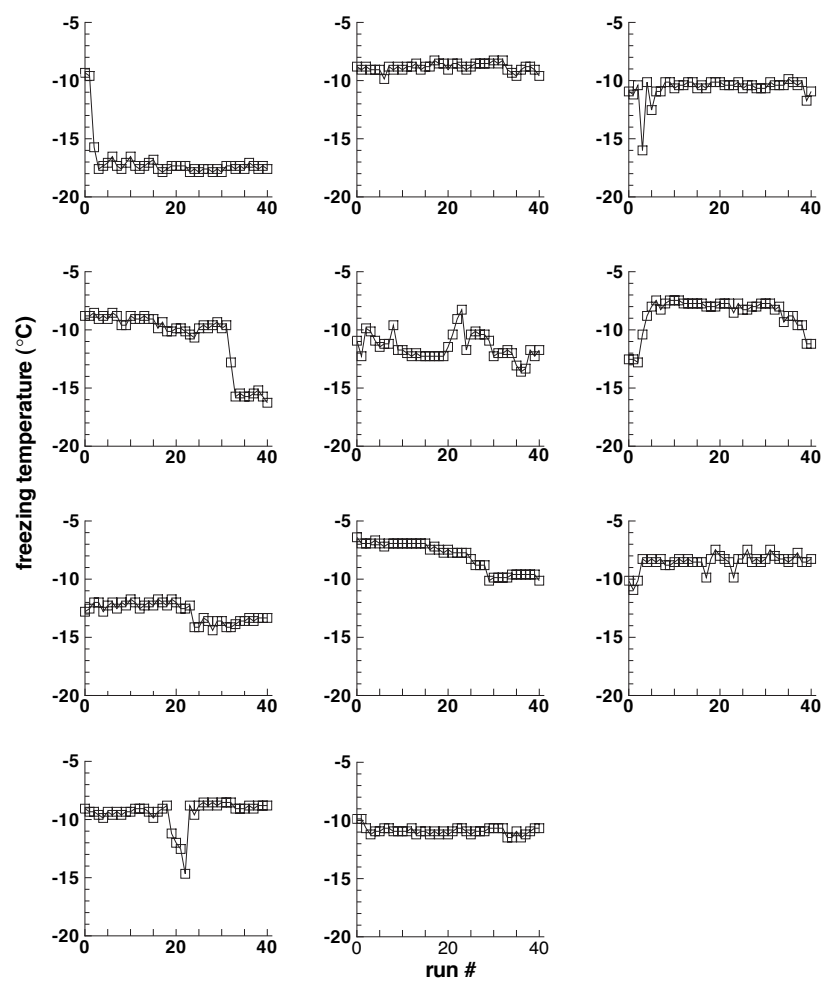

Fig. 4. Examples of drop histories from the SN subset of soil sample A over 41 consecutive runs.

runs. Due to partial evaporation of the drops, some distilled water was added to bring them back to original volume at the beginning of the third day. This was done to facilitate detection of freezing; the distilled water contained no nuclei active in the range observed for the soil suspension.

The main features of the observations with this sample are summarized in Fig. 5. The average freezing temperatures rose over the first 10 runs and then decreased until the end of the sequence. Overall variations are greater than those found with sample A, even though run-to-run changes (ignoring the jump accompanying the overnight change after run \#42) are comparable. The average of the absolute value of the run-torun changes in the average freezing temperature is $0.083^{\circ} \mathrm{C}$ which is in fact a bit smaller than the $0.096^{\circ} \mathrm{C}$ value for sample A. The difference lies in the regularity of the changes for this sample versus the more erratic pattern for A. The rank correlation coefficients shown in the lower panel of Fig. 5 have low values at the overnight gaps (indicated by dashed lines). However, even these values have roughly $10^{-21}$ probability to have been due to chance. The average, excluding the two overnight values, is 0.92 .

The SN subset for sample B was selected for runs \#19 to \#42 and for 56 drops with $T_{s n}>-13.5^{\circ} \mathrm{C}$. The average rank correlation coefficient for this set is 0.93 . The contour plot of the frequency of run-to-run changes in freezing temperatures for this group of data is very much like that shown in Fig. 2a. (a)

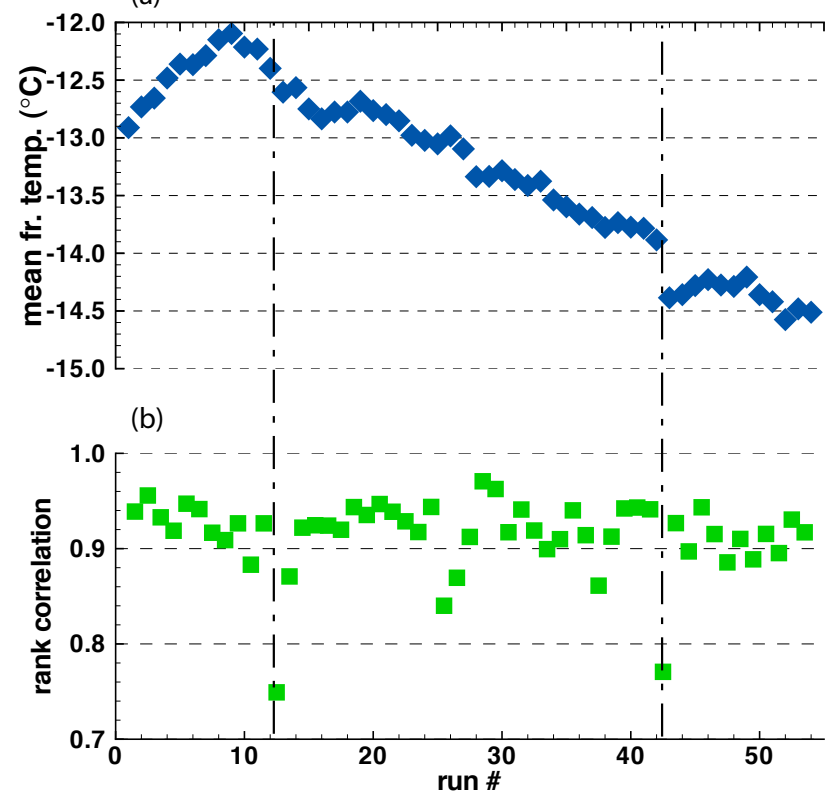

Fig. 5. Average temperatures (a), and rank correlation coefficients (b) for soil sample B. The dashed vertical lines indicate the two overnight gaps (about $15 \mathrm{~h}$ each).

\subsection{Distilled water}

In order to extend the range of temperatures of the tests, a sequence of runs was made with distilled water. This is the same water that was used to prepare the soil suspensions. It originated from a glass laboratory still of average quality and was kept in a large bottle for use over long periods of time. For the re-freeze experiments 78 drops were used and a total of 40 runs were made. There was an overnight break after run \#10.

Freezing temperatures for this sample ranges from $-10^{\circ} \mathrm{C}$ to $-24^{\circ} \mathrm{C}$ with the majority of the events between $-16^{\circ} \mathrm{C}$ and $-22^{\circ} \mathrm{C}$. During the 40 runs, the mean temperature drifted to higher values at first then gradually decreased, as shown in Fig. 6. The absolute values of the changes averages $0.1{ }^{\circ} \mathrm{C}$, not significantly different from what was found for the soil samples. Neither is the degree of repeatability between runs, as the mean rank correlation coefficient is 0.91 with a significance level of the order of $10^{-9}$. Table 2 (Row 1) shows the pdf of the $\delta T$-values for the entire sample and for the SN subset. The latter was defined for runs \#14 to \#40 to focus attention of the portion of the sequence with steady changes. The cutoff temperature based on run $\# 14$ was $T_{s n}=-18.7^{\circ} \mathrm{C}$; the resulting sample size is 35 drops. Results for the SN subset are shown in Row 2 of Table 2, and in Fig. 7; in general, these are quite similar to results obtained with the soil samples. 
Table 2. Distribution of run-to-run temperature changes, $\delta T_{i j}\left({ }^{\circ} \mathrm{C}\right)$ for distilled water.

\begin{tabular}{llccccccccccccc}
\hline Row & Data & \multicolumn{10}{c}{ Percentile } \\
\cline { 3 - 11 } & & 0.1 & 0.5 & 1 & 5 & 10 & 25 & 50 & 75 & 90 & 95 & 99 & 99.5 & 99.9 \\
\hline 1 & Runs \#1..440 (3042 values) & -4.8 & -3.2 & -2.7 & -1.3 & -1.1 & -0.54 & 0 & 0.54 & 0.81 & 1.3 & 2.7 & 3.8 & 5.1 \\
2 & SN subset (910 values) & & -2.8 & -2.4 & -1.3 & -0.81 & -0.4 & 0 & 0.27 & 0.81 & 1.1 & 2.3 & 2.8 & \\
\hline
\end{tabular}

(a)
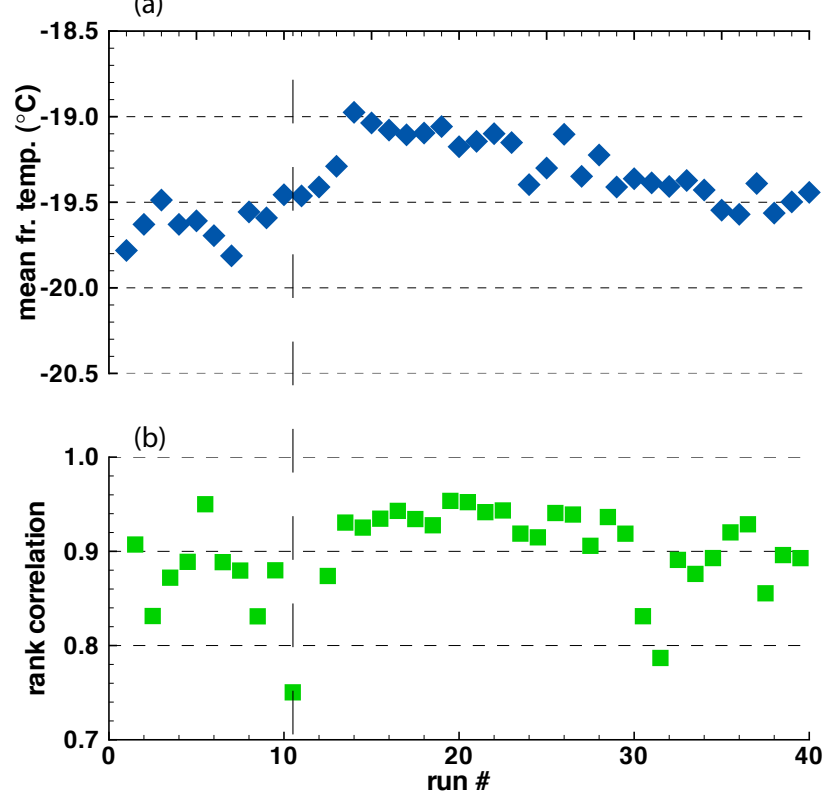

Fig. 6. Average freezing temperatures (a), and rank correlation coefficient (b) for 40 runs with distilled water. The vertical dashed line indicates a 15 -h pause in the experiment.

A rough comparison was also made between the results described above and those for the 48 drops of distilled water included in the experiments with soil sample A. To avoid excessive detail, results from those runs are not included here. In general, no differences worth noting have been seen between the two sets of experiments.

\section{Analysis and interpretation}

The distinction between random and systematic changes in the data is not as easy to draw as one would like. It has to be done at two levels. First, it is relatively obvious from the data that a fully random rearrangement of freezing temperatures among drops from a given sample is incompatible with the observations. The contradiction with a stochastic description is clearly demonstrated by the contrast (described in Sect. 5.1) between the observed correlation of 0.98 versus the near zero value for randomized temperatures of the second of a pair of runs. In the same vein, the distribution of (a)
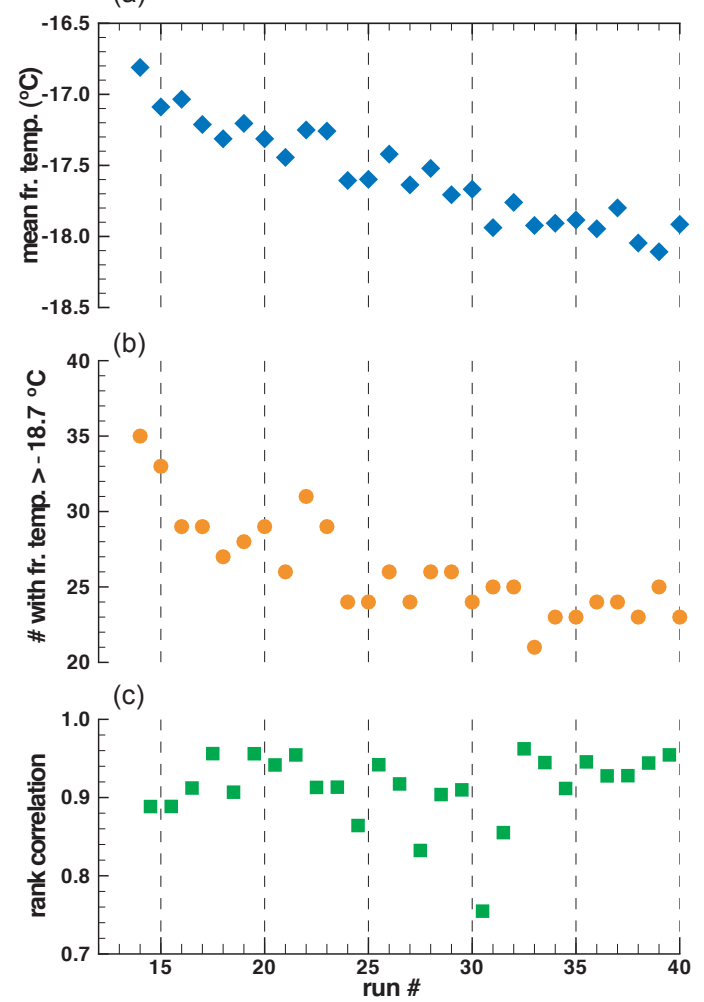

(d)

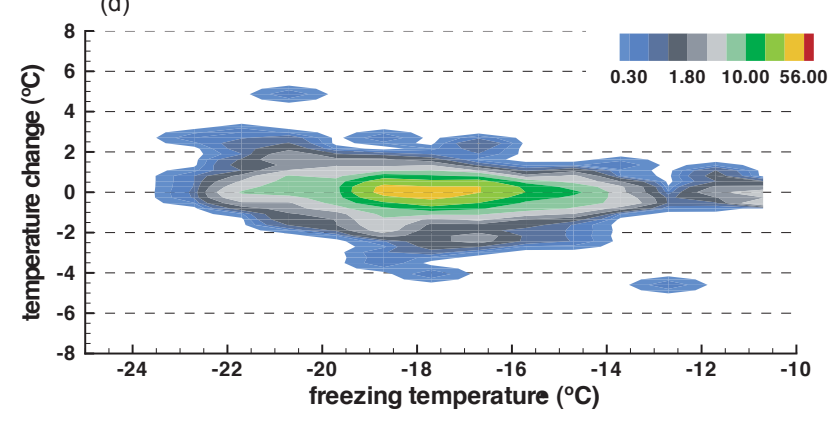

Fig. 7. Results for the SN subset of the distilled water sample. (a) Average freezing temperatures; (b) the number of drops with freezing temperatures above the SN cutoff. (c) Rank correlation coefficients. (d) Contour plot of the frequencies of the observed changes in freezing temperatures. The colored density scale is logarithmic, and is in units of $0.27\left({ }^{\circ} \mathrm{C}\right)^{2}$. 
(a) Soil sample A

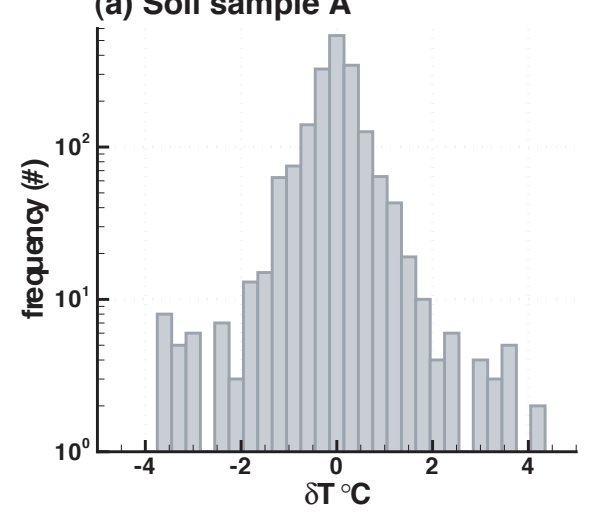

(b) Soil sample B

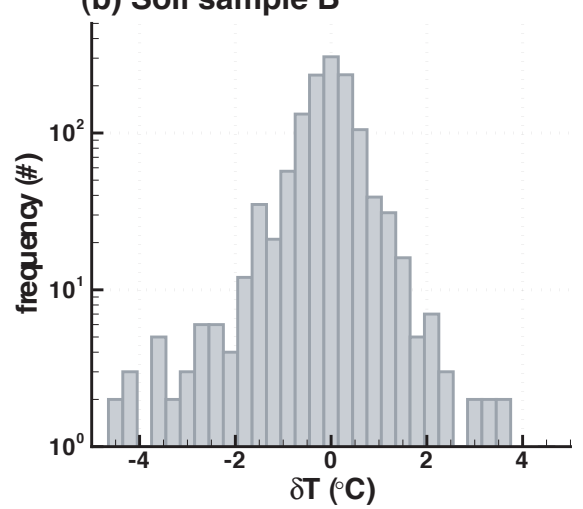

(c) Distilled water

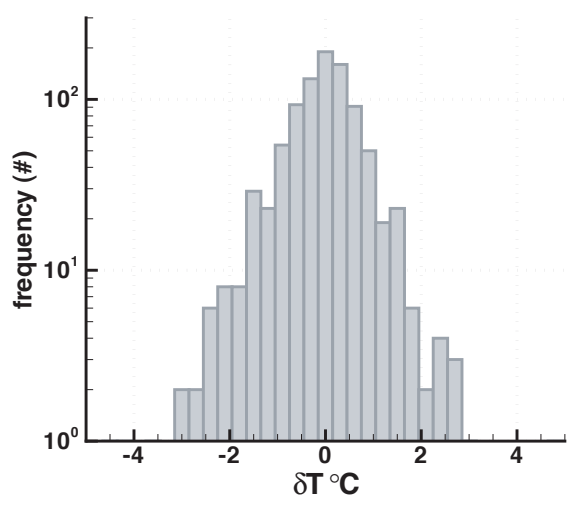

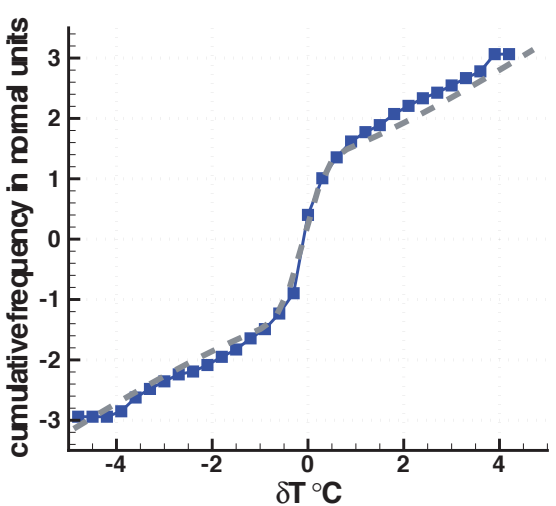
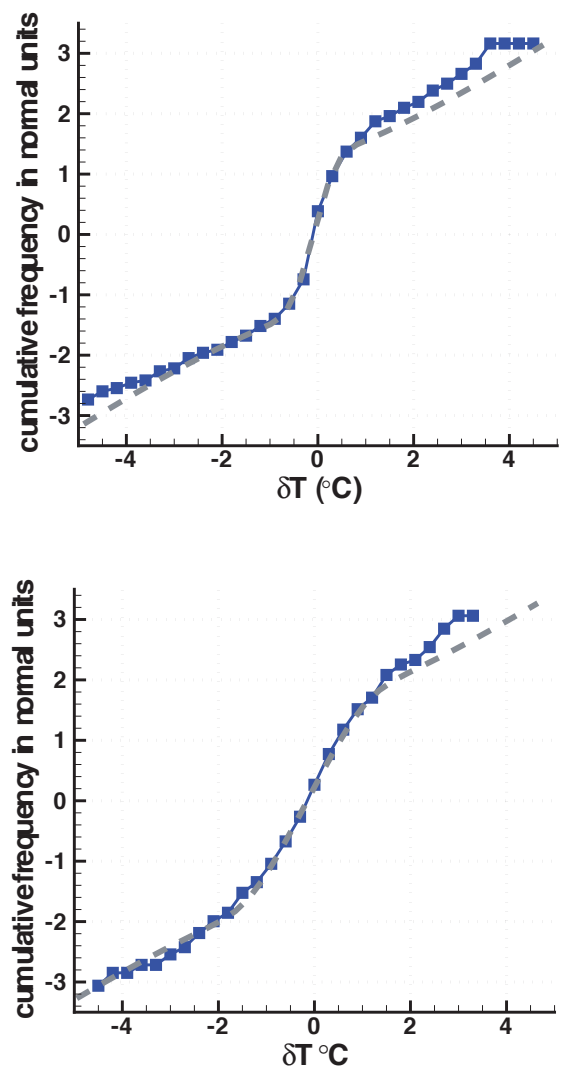

Fig. 8. Histograms and cumulative frequency distributions of run-to-run temperature changes for the SN subsets of (a) Soil sample A, (b) Soil sample B, and (c) distilled water sample. The ordinate in the left-hand panels is the frequency of freezing events per $0.3^{\circ} \mathrm{C}$ temperature intervals. The right-hand plots are on probability paper, the ordinate being labelled in units of standard deviations of a normal distribution. In the cumulative plots, the dashed lines show the sum of two normal distributions fitted to the data.

$\delta T$-values has been looked at for 100 re-randomizations of observed freezing temperatures (using run \#26 of sample A). The results are listed in Row 3 of Table 1; a comparison of those numbers with values in Rows 1 and 2 of Table 1 clearly show that random variations of the freezing temperatures, even when restricted to the range actually observed, would lead to run-to-run changes much larger (up to 10 times for the central part of the distribution) than observed.
The opposite assumption, the singular description, has convincingly strong support from the high values of the rank correlation coefficients and the infinitesimally small probabilities that those values arose by chance. The problem is that it is also clear that singularity is only valid to a first approximation. That additional factors need to be considered is evident from a number of observational facts: (i) The individual histories of drops are quite varied, as shown in Fig. 4. (ii) 
The mean freezing temperatures of the populations of drops for any given sample do not remain constant during the refreezing sequences. (iii) The correlation coefficients (either in terms of temperatures or as ranks) are high but not equal to unity, and at times are significantly lower. The combined effects of these facts are apparent when the pdf of $\delta T_{i j}$ is examined in detail. Figure 8 shows these distributions for the SN subsets of the three samples discussed in Sect. 5. Pulling together all values in these plots is not unreasonable, since the dependence of $\delta T$ on $T$ isn't strong, as can be seen in Figs. $2 \mathrm{a}$ and $7 \mathrm{~d}$, although more will be said about that later.

The left-hand panels in Fig. 8 are straightforward representations of the raw data. The main new finding is represented in the right-hand panels. Here the cumulative frequencies of $\delta T$ are plotted with the ordinate in normal units, i.e. as multiples of unity standard deviation, so that a Gaussian pdf would appear as a straight line in the graph. The observed distributions (blue squares) do not follow that pattern but are strongly S-shaped. That shape suggests that the data may be approximated by the weighted sum of two normal distributions. For Fig. 8a and b, the broken lines show the sum of two distributions, one with $\sigma_{1}=0.28^{\circ} \mathrm{C}$ and $w_{1}=0.78$ and the other with $\sigma_{2}=1.8^{\circ} \mathrm{C}$ and $w_{2}=0.22$. The corresponding values for Fig. $8 \mathrm{c}$ are $\sigma_{1}=0.6^{\circ} \mathrm{C}, w_{1}=0.86, \sigma_{2}=1.8^{\circ} \mathrm{C}$ and $w_{2}=0.14$. All these were determined by trial and error and the fit was adjusted by eye. While the separation of the observed pdf into two components is a somewhat arbitrary step, the drop histories in Fig. 4 also suggest that they consist of relatively stable segments interrupted or terminated by more erratic-looking behavior.

Coupled with the basically singular character of freezing nucleation, the decomposition of the observed frequency distributions parallels the suggestion from the individual drop histories that the changes in freezing temperatures can be thought of as being of two kinds: "fluctuations" and "nonrandom alterations". By fluctuations we refer to the relatively small changes which appear random and are then characterized appropriately by the narrower of the two normal distributions. Alterations are left to encompass other changes that follow non-random patterns like the drifts, jumps, slow irregular variations, etc. that are seen in the drop histories. That these variations are approximated by a second normal distribution is only a convenient description with no fundamental reason to follow that mathematical function. For some of these larger changes it is not possible to distinguish between alterations of a particular site or activity by another site altogether. It is also apparent that these alterations can represent systematic trends over several runs, that is over hours.

In the terms of the definitions of the preceding paragraph, the model that emerges is that two additional factors have to be added to the singular description. The characteristic temperature of a nucleating site determines, at a given time and as a first approximation, the temperature at which nucleation can be expected to occur on it. Randomness, associated with molecular dynamics, adds a fraction of a degree variability.
Thirdly, slow alterations, whose possible causes are to be discussed later, may lead to changes of up to several degrees and have to be considered a change in the characteristic temperature itself. It is important to note that the third element of the model is absent for the majority of cases; the values of $w_{2}$ quoted in the preceding paragraph suggest that it comes into play about a fifth of the time, but this will need further examination.

\subsection{Nucleation rate}

It appears clear that interpretation of the observations here presented in terms of the classical nucleation theory would require a large number of assumptions to be made in order to explain the range of freezing temperatures found for any given sample and to deal with the fact that the nuclei involved in these experiments are mixtures of many substances. Nonetheless, the evidence for fluctuations described in the preceding section can be used to derive the form of the nucleation rate function. Nucleation rate is derived from experiments with repeated freezing of a sample (drop) as $J=(1 / A \times N)(d N / d t)$, i.e. the fraction of unfrozen drops freezing per unit time normalized to unit surface area $(A)$ of the nucleating substance in the sample.

To calculate $J$ from the data here presented, the first step is to accept the characterization of the fluctuations, captured with the value of $\sigma_{1}$, as what would have been observed in the absence of the additional alterations, and that it is valid for individual nuclei or sites. In support of that association, it is relevant to mention that $50 \%$ of the sequences which have $\delta T_{i j}<0.6^{\circ} \mathrm{C}$ are 13 runs long and that for $\left|\delta T_{i j}\right|<1.0^{\circ} \mathrm{C}$ they are 26 runs long (for sample A). Visual inspection of the drop histories (Fig. 4, and Supplement) lends further support.

With the aforesaid caveat, the nucleation rate $J$ is determined from the fraction of drops freezing per temperature interval for a normal distribution width $0.71 \times \sigma_{1}$. The factor 0.71 arises from the fact that $\sigma_{1}$ represents the distribution of changes, not of the deviations from the mean. Simple simulations indicate that if one of these distributions is normal, so is the other, and that the widths of the two distributions have a ratio of 0.71 . Gaussian distributions of freezing frequencies with $\sigma=0.71 \times \sigma_{1}$ corresponding to the soil suspension $\left(\sigma_{1}=0.28\right)$ and to the distilled water $\left(\sigma_{1}=0.6\right)$ samples are shown in the left-hand and middle panels of Fig. 9. The nucleation rates corresponding to those distributions are shown in the right-hand panel of Fig. 9. Since the surface area of nucleating substance per drop is not known for these samples, the rate is given relative to an arbitrary value of unity at the characteristic temperature $T_{c}$. The abscissa, $\Delta T$, is the deviation in temperature from $T_{c}$. As seen in other expressions of the nucleation rate, that rate rises quite abruptly as the critical value (in this case $T_{c}$ ) is approached.

The nucleation rate shown in Fig. 9 refers to a particular nucleus or site defined by its characteristic temperature. Representing the nucleation rate in this manner, and not as 

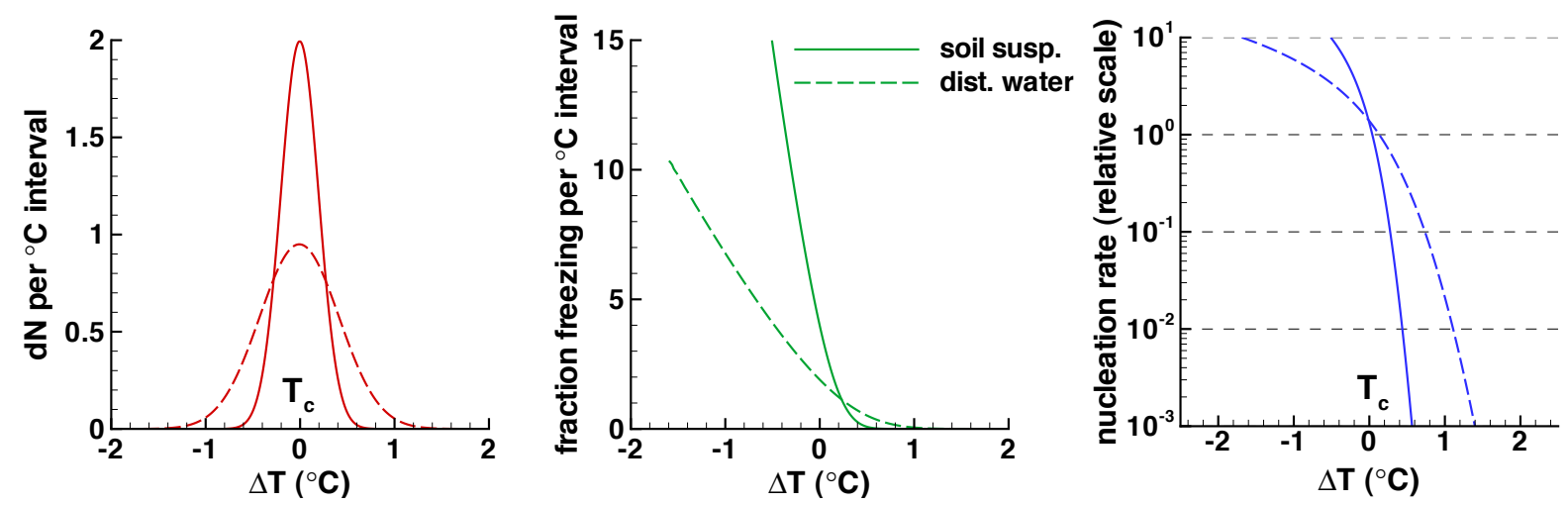

Fig. 9. Gaussian pdf with $\sigma=0.2$ (full line) and $\sigma=0.42$ (dashed line) corresponding to the number of times freezing would occur (in an infinite sequence of repeated trials) as a function of the deviation of temperature from the characteristic temperature (left panel). The fraction of unfrozen drops freezing within unit temperature interval for the distributions shown in the left-hand panel (middle panel), and the latter expressed as nucleation rate on a relative scale (right-hand panel).

a function of specific temperatures for a specific substance as is usually done, is an important aspect of the composite model being put forward in this paper. Thus, it is not suitable for quantitative comparisons with classical nucleation theory. Neither is it clear what area should be considered, in principle, to give and absolute value of the rate. Total surface area of the substance in the sample (per drop in this type of experiment) is appropriate when known amounts of a single substance are involved. That concept breaks down when applying it to a particular nucleating site. It is better to think of the nucleation rate as an expression of the probability that nucleation will take place on that site within unit time, as a function of the temperature deviation from $T_{c}$. In that sense, the right-hand panel of Fig. 9 is a quantification of Fig. 12 in Vali and Stansbury (1966); this is the first time that this has been achieved. In addition to this probability one should consider the probability that a site with given $T_{c}$ occurs in a sample. Equations (1) and (2) provide one from of expressing that, while variations of those formulas can, in principle at least, readily be constructed to normalize the overall probabilities to the amount of solid material in the suspension, or other desired references.

The rate functions in Fig. 9 are in general steeper than those reported by Seeley and Seidler (2001a, b) and by Zobrist et al. (2007) for alcohol monolayers. The main reasons for the difference in slope is that the rate in Fig. 9 is based on isolating one type of variation from the data while the references cited use direct observations for a small number of droplets. Differences can also be expected due to the use of different nucleating substances in the experiments. Whether the flatter rate curve for distilled water in comparison with the soil sample is significant, or not, and what that difference might indicate will remain for further work to determine. Since the freezing temperatures of the distilled water sample were about $5^{\circ} \mathrm{C}$ colder than for the soil suspension, the opposite trend could have been expected from the smaller critical embryo size required.

The combined effects of the nucleus-specific characteristic temperature and the time-dependence that arises from the appreciable variations of the nucleation rate can be effectively seen in the results of experiments in which 20 re-freezing cycles were carried out with cooling rates alternating between two different values: 0.7 and $4.0^{\circ} \mathrm{C} \mathrm{min}^{-1}$. Due to a mishap, data are available only for 11 drops but those drops had initial freezing temperatures ranging between $-12^{\circ} \mathrm{C}$ and $-24^{\circ} \mathrm{C}$ so that fairly definite results can be extracted from the data in spite of the small numbers of drops. The findings of importance for the discussion here are that (i) the mean freezing temperature at the higher cooling rate was $0.4^{\circ} \mathrm{C}$ lower than in the slow runs, similarly to results in Vali and Stansbury (1966), and (ii) that the rank correlations between runs of differing cooling rates was the same as between runs of the same rate. These features are illustrated with the drop histories shown in Fig. 10. The systematic temperature difference as well as the parallel variation between the two parts of the sequence are clearly evident. More details of the results are included in the Supplement. These observations clearly support the singularity of freezing temperatures. The $0.4^{\circ} \mathrm{C}$ shift associated with the roughly factor 6 increase in cooling rate is quite compatible with the variations in nucleation rate shown in Fig. 9. Since the quantitative estimate for the range over which $J$ varies for a given nucleus is one of the principal new results from this work, it is reassuring to have support for Fig. 9, even if it is only in the sense of consistency.

\subsection{Alterations of $T_{c}$}

The second, non-random, component of the overall variability in freezing temperatures over repeated trials with a given nucleus is termed "alterations". There is a very large body of 

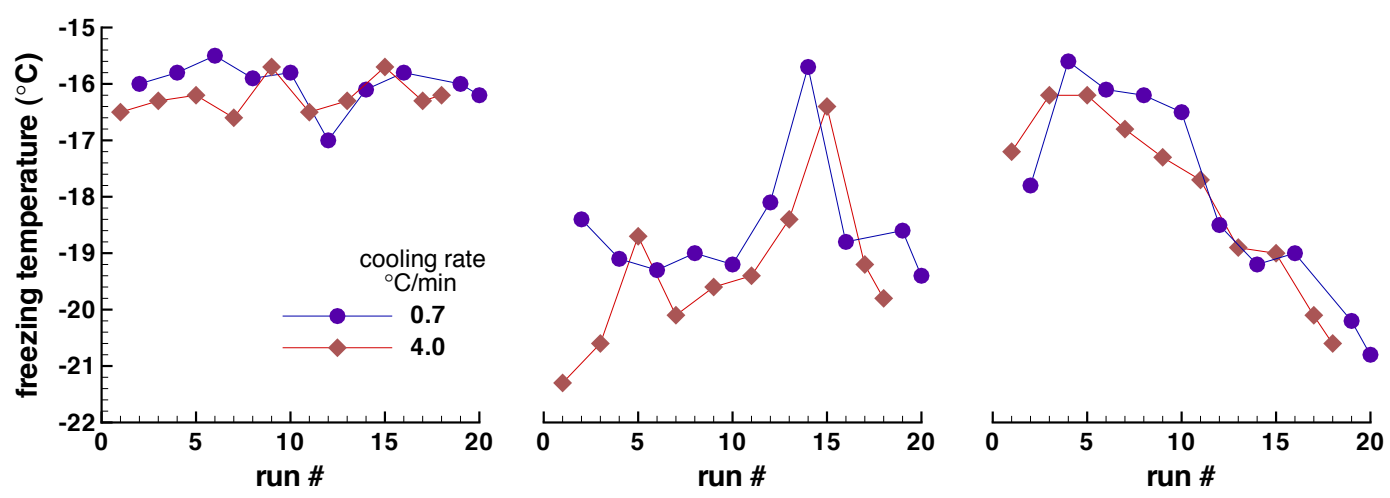

Fig. 10. Examples of freezing temperatures observed with three different drops when the rate of cooling was alternated between two values. At the end of the sequence two runs each were made with the two different cooling rates.

literature dealing with "pre-activation", "de-activation" and with "memory effects" in heterogeneous ice nucleation ${ }^{5}$ (and in other phase changes); the word alterations encompasses those phenomena but is intended to be broader. Perhaps the principal distinguishing feature of these changes from random fluctuations is that they appear to depend on the prior history of nucleation events undergone by the sample, for example whether an early part of a sequence is considered, or a later one.

Definition of the characteristic temperature $T_{c}$ as the "value at which nucleation is most likely to occur" was made anticipating the need to deal with these alterations apart from the time-dependent effects associated with the nucleation rate. However, that definition carries with it the broad general assumption that nucleation sites on a substrate are specific surface features either crystallographically, or in composition.

As indicated earlier, alterations are observed in about $15 \%$ of the re-freezing cycles and result in changes of freezing temperatures with a standard deviation of about $1.8^{\circ} \mathrm{C}$. These are rather large changes compared with what results from random fluctuations. The experiments provide only a diagnosis of these alterations and interpretation of what causes them can only be speculative at this point. The observations do provide information, beyond the range of magnitudes, on the time scales and patterns of these changes. As a rough descriptive classification one can consider the following: a) drifts, or monotonic changes over time periods of tens of minutes to hours, b) short-term spikes, (either positive or negative): changes greater than one degree over one or two runs followed by a return to the original value, c) oneway jumps from one level to another, and d) meanderings, which are changes similarly random appearing but of greater magnitude than the fluctuations discussed in Sect. 6.1. This classification is not meant to be rigorous by any means, but

\footnotetext{
${ }^{5}$ Comparisons of our observations with those reports would add too much to the length of this paper, so it will be left for another paper.
}

only a basis for speculating about processes that may be responsible for them. Examples of these alterations, and combinations of them can be seen in Figs. 4 and 10, with further examples given in the Supplement.

In qualitative terms, possible reasons for the positive changes (raising the freezing temperature) include retention of small ice clusters on the sites, imprinting the ice configuration on the substrate, solution effects, or etching of the substrate surface. Negative changes may be caused by some stressing or partial destruction of sites, contamination by foreign matter, or the retention of some molecular clusters in less than optimum configuration for ice embryo growth. Movement of the a nucleus from the interior of the drop to the air-water interface may also lead to changes, as reported by Shaw, Durant and Mi (2005) and Durant and Shaw (2005).

One manifestation of the alterations can be seen in the gradual changes of average freezing temperatures in all of the tests. Perhaps surprisingly, a rise in the average was found at the beginning of some sequences; very clearly in Fig. 5 and somewhat less clearly and actually not at the very beginning in Fig. 6. Since these changes, in either direction, represent gradual drifts for the large majority of drops, not large changes in a few, they are perhaps the most important. This adds an important constraint as to which of the possible explanations listed in the preceding paragraph may be at work, but it is not clear which one. The same can be considered to apply to individual drops undergoing gradual drifts. At the other extreme, the larger jumps are more compatible with the possibility of total deactivation of one site and a change to activity by another of higher or lower $T_{c}$. Similarly, spikes could indicate a temporary loss of a site and another becoming the determinant one.

The alterations here discussed were observed with nuclei that have been in suspension for hours to days. Therefore, it remains to be determined what is the importance of these findings to atmospheric processes which are generally much faster. There are additional factors to consider in assessing relevance to atmospheric processes. The composition of the 
nuclei in these experiments was not determined, however the soil samples do represent potential sources of atmospheric aerosols. Also, the samples contained undetermined amounts of dissolved substances, though the concentration of solutes was almost certainly below 0.1 molal, as judged from the onset of melting at $0^{\circ} \mathrm{C}$. Overall, on the one hand, one could argue that there is very little relevance of these alterations to ice formation in clouds because of the differences in time scales. On the other hand, the alterations found in this work could indicate that faster and perhaps even larger changes may occur when particles are first immersed in water by condensation or capture; in that case the prediction of ice initiation in clouds becomes an even more difficult goal, either from the measurement or theoretical points of view. These uncertainties in making the connection between the laboratory experiment and cloud processes could be narrowed considerably by performing further experiments with nuclei of known composition and size, and with water containing different solutes.

\subsection{Comparisons with other results}

As mentioned in the introduction, many previous studies overlap with the topic of this paper. Here, only a few specific parallels and divergences will be highlighted. All of these experiments involved subjecting samples to repeated freezing cycles.

The extensive data set of Dorsey $(1938,1948)$ contains many identical features to our data. From his first observations, he concluded that " $\ldots$ the extent of supercooling of a given specimen of distilled water at a given time is fixed within moderately narrow limits, [and] varies from specimen to specimen ...". These conclusions were later extended to samples with various types of nuclei. The samples were $6 \ldots 8 \mathrm{~cm}^{3}$ in volume and were contained in carefully cleaned and sealed glass bulbs. Tests were made both on the scale of hours (1948, Table 3), and at intervals of many months. The sequences of freezing temperatures reported for individual samples show relatively constant segments, and the kinds of alterations discussed in Sect. 6.2. Much of Dorsey's concern was centered on these alterations and possible reasons for them, including dilution, mixing of two samples, heating, and so on. In addition to the conclusion cited above, he found that each sample had several preferred temperatures of freezing. No effect of prolonged chilling was detected. Random effects were not considered.

Bayardelle (1954) reported observing both slow variations and rapid fluctuations between cycles with water drops suspended at a mercury-silicon oil interface and concluded that the stochastic theory was incompatible with the results. Carte (1956) too found that freezing temperatures may stay constant over hundreds of repetitions but that slow drifts occurred in a small fraction of cases. Alternate cooling rates were accompanied by a $0.5^{\circ} \mathrm{C}$ difference, much like the findings in this work.
Vonnegut and Baldwin (1984) conducted tests by exposing a silver iodide suspension many hundreds of times to the same temperature and recording the length of time until freezing occurred. The average time to freezing decreased from a few seconds at $-9^{\circ} \mathrm{C}$ to near $3 \mathrm{~h}$ at $-3^{\circ} \mathrm{C}$, and at any given temperature the lapse of time had a negative exponential distribution. Some systematic changes were also observed and associated with possible surface changes of the silver iodide due to mechanical effects of freezing. The authors equate the results of many tests with one sample to what would be expected for a large number of identical samples in a single test. That assumption is valid for truly identical samples, but it may not hold for macroscopically similar samples if the most active sites differ, which is actually likely for sites that have a low probability of being found in a sample. As shown by Vali (1994) the results are consistent with the pdf of expected times needed for nucleation at a temperature where the nucleation rate is low, i.e. above the characteristic temperature of the most active particle, or site, in the particular suspension that was tested. The finding of at least one period of different behavior for the sequence for which detailed data are presented in the paper also supports that interpretation by indicating the importance of specific sites. The diminution of lapse times for colder temperatures indicates a $10^{4}$ increase in nucleation rate for $6^{\circ} \mathrm{C}$ lower temperature.

In the work of Zobrist et al. (2007; Z07), individual drops were subjected to re-freezing cycles with cooling rates of $10^{\circ} \mathrm{C} \mathrm{m^{-1 }}$. with an emphasis on testing the activity of nonadecanol coatings on the drops. In a control test, an uncoated drop of comparable size to those used in our experiments was found to freeze near $-20^{\circ} \mathrm{C}$ with only small changes in freezing temperatures in nearly 100 cycles. Nucleation is assumed to be initiated by the supporting surface. The nucleation rate (Z07, Fig. 2) has a slope of about factor 10 per $0.7^{\circ} \mathrm{C}$, a value close to that shown in Fig. 9 for distilled water near $T_{c}$. For this drop, the observations are comparable with those reported in this paper, and the results are consistent with the modified singular model presented here. For drops coated with nonadecanol, freezing temperatures exhibited significantly larger run-to-run variability: a range of nearly ten degrees for drops of the same size as the uncoated sample and a range of a few degrees for drops of near $40 \mu \mathrm{m}$ diameter. Consequently, the derived nucleation rate for the larger drops shows a temperature dependence lower by about a factor of five than for the uncoated drop and the rate for the smaller drops is intermediate between those values. To interpret these data in terms of each observed freezing event being due to the most active nucleating site in the sample, one would have to view the nonadecanol layer as having very few sites and that the probability of nucleation on those sites increases only slowly as the temperature is lowered. That behavior would be radically different from what we have found for other materials. 
In Z07, the observations are interpreted in terms of the stochastic model. The derived nucleation rate over a temperature range of nearly $20^{\circ} \mathrm{C}$ is reconciled with CNT by allowing the effective contact angle to vary by a factor of two over that range. This description of the process can be viewed as indication that nucleation sites form within the nonadecanol monolayer in a random fashion during the cooling process. While there is no evidence to the contrary, this scenario is difficult to reconcile with the activity of nonadecanol at relatively small supercoolings, and with the fact that it has a crystalline structure, albeit it is known that some rearrangements may take place in it as cooling progresses. Alternatively, stochastic behavior could arise from the presence of a very large number of nearly identical sites, each with a probability of freezing that increases slowly with decreasing temperature. This latter scenario would lead to observations essentially indistinguishable from the predictions of the singular model and also requires that the nucleation probability have a weak dependence on temperature. Such a behavior is contrary to the steep rise in nucleation rate that is the generally observed for both homogeneoous and for heterogeneous freezing; it also differs from the measurements of Seeley and Seidler (2001a) with other alcohol monolayers.

The applicability of a stochastic description can be tested by demonstrating that the nucleation rate is independent of the time-temperature relationship in the experiment, i.e. by varying the rate of cooling, or, more effectively yet, by comparing the results obtained with continuous cooling with those observed at constant temperatures. In Fig. 3 of Z07, one data point is presented that is derived from three measurements of the time till nucleation at constant temperature with the same drop. While this data point is set apart somewhat from the points obtained with constant cooling, the derived rate curve for CNT fits this point as well. In all, support provided by this one data point for the stochastic model must be considered somewhat weak, and it seems reasonable to say that a more definite conclusion about which model is more appropriate to nucleation by nonadecanol coatings will have to await future experiments.

Marcolli et al. (2007), working with suspensions of Arizona Test Dust in a differential scanning calorimeter, found good agreement with the singular model. Their observations were well approximated by assuming that the probability of occurrence of an active site per unit surface area is a lognomal function of contact angle. Contact angle is used as a surrogate for activity at various temperatures, and parallels the role of characteristic temperature $T_{c}$; the probability function they assumed fills the same role as the nucleus spectrum shown in Fig. 1c which has an empirical basis. The stochastic variation remains a superimposed effect in both sets of observations.

\section{Summary}

Experiments were described in which roughly 100 drops were subjected to simultaneous cycles of freezing and melting. The drops contained suspended foreign matter and had nucleation temperatures between $-6^{\circ} \mathrm{C}$ and $-24^{\circ} \mathrm{C}$. Cycle times were of the order $15 \ldots 20 \mathrm{~min}$ and also included some gaps of about $15 \mathrm{~h}$.

Results derived from these experiments were used to distinguish between random variations of nucleation temperatures that are inherent in the nucleation process and more systematic alterations of the nuclei that accompany a minority of the events. Both of those effects are superimposed on the characteristic nucleation temperature, $T_{c}$, that is specific to each particular nucleus, or site. Thus, the results provide confirmation and extension of the modified singular description of heterogeneous freezing nucleation (Dorsey, 1948; Vali and Stansbury, 1966; Vali, 1994) and delineate the extent to which randomness due to molecular fluctuations modify that model.

Random variations in nucleation temperatures (for the cooling rate applied) were found to be characterized by standard deviations of $0.2^{\circ} \mathrm{C}$ for the soil samples and $0.42^{\circ} \mathrm{C}$ for the distilled water sample. These deviations from $T_{c}$ were seen in roughly $85 \%$ of the cases. No clear variation of the width of the distributions with temperature could be detected for either sample over ranges of about $8^{\circ} \mathrm{C}$. It remains to be established by further work whether the type of nuclei (composition, etc.) lead to systematically different widths; comparisons with Seeley and Seidler (2001a, b) and Zobrist et al. (2007) suggest that that may be the case. Nucleation rates were derived as functions of the temperature deviation $\Delta T$ from $T_{c}$, as shown in Fig. 9. This is an important deviation from the usual view that nucleation rate can be defined as characteristic for a given substance and that it can be evaluated in terms of the bulk properties of the material.

The third component observed to affect nucleation temperatures in these experiments was termed "alterations" as they appear to depend on the prior history of the drops, have rather specific variants and have systematic components. These alterations provide some hints about processes influencing the activity of nuclei and they are of importance in interpreting experiments involving repeated freezing of samples.

Based on these results, models of ice initiation in clouds via immersion freezing can be considered at three levels. First, predictions should be based on a pdf of the $T_{c}$-values and the distribution of the nuclei in cloud droplets by condensation or by capture. For cloud volumes cooling at rates from, say, a tenth to the full adiabatic rate that is going to be an adequate predictor, certainly well within the accuracies with which those pdf can be determined either by direct measurement or by some generalized formula. The acquisition of sufficient empirical data to reliably define these pdf remains the main challenge. Second, for slower rates of cooling, or for constant temperatures, the nucleation rate for given $T_{c}$ 
needs to be considered: rates roughly like those in Fig. 9 provide a usable approximation until the dependence of the rate curve on particle composition is better known. Third, the systematic, though unpredictable, alterations here reported need not be a concern for cloud models, except for special cases where cycling of drops through freezing and melting is expected.

Acknowledgements. Partial support for this work was derived from grant ATM-0650609 from the US National Science Foundation, Physical and Dynamic Meteorology Program. Insightful comments by two reviewers were very helpful in improving the paper.

Edited by: T. Koop

\section{References}

Bayardelle, M.: Sur le mecanisme de la congelation de l'eau dans les nuages, Compt. Rend. Acad. Sci., 239(16), 988-989, 1954.

Bigg, E. K.: The supercooling of water, Proc. Phys. Soc., B 66, 688-694, 1953.

Carte, A. E.: Probability of freezing, Proc. Phys. Soc., B 73, 324, 1959.

Carte, A. E.: The freezing of water droplets, Proc. Phys. Soc., B 69(10), 1028-1037, 1956.

Diehl, K. and Wurzler, S.: Heterogeneous drop freezing in the immersion mode: Model calculations considering soluble and insoluble particles in the drops, J. Atmos. Sci., 61(16), 2063-2072, 2004.

Dorsey, N. E.: Supercooling and freezing of water, J. Res. Natl. Bureau Standards, 20(6), 799-808, 1938.

Dorsey, N. E.: The freezing of supercooled water, Trans. Amer. Phil. Soc., 38(3), 248-328, 1948.

Dufour, L. and Defay, R.: Thermodynamics of clouds. Academic Press, New York, 255 pp., 1963.

Durant, A. J. and Shaw, R. A.: Evaporation freezing by contact nucleation inside-out, Geophys. Res. Lett., 32, L20814, doi:10.1029/2005GL024175, 2005.

Fletcher, N. H.: The Chemical Physics of Ice, University Press, Cambridge., 271 pp., 1970.

Kärcher, B. and Lohmann, U.: A parameterization of cirrus cloud formation: Heterogeneous freezing, J. Geophys. Res.-Atmos., 108(D14), 4402, doi:10.1029/2002JD003220, 2003.

Khvorostyanov, V. I. and Curry, J. A.: The theory of ice nucleation by heterogeneous freezing of deliquescent mixed CCN. Part II: Parcel model simulation, J. Atmos. Sci., 62(2), 261-285, 2005.

Langham, E. J. and Mason, B. J.: The heterogeneous and homogeneous nucleation of supercooled water, Proc. Roy. Soc. A., 247, 493-505, 1958.
Levin, J.: Statistical explanation of spontaneous freezing of water droplet, Natl. Adv. Comm. Aeronautics, Techn. Note 2234, 27 pp, 1950.

Liu, X. H. and Penner, J. E.: Ice nucleation parameterization for global models, Meteorol. Zeitsch., 14(4), 499-514, 2005.

Marcolli, C., Gedamke, S., Peter, T., and Zobrist, B.: Efficiency of immersion mode ice nucleation on surrogates of mineral dust, Atmos. Chem. Phys., 7, 5081-5091, 2007, http://www.atmos-chem-phys.net/7/5081/2007/.

Morrison, H., Shupe, M. D., Pinto, J. O., and Curry, J. A.: Possible roles of ice nucleation mode and ice nuclei depletion in the extended lifetime of Arctic mixed-phase clouds, Geophys. Res. Lett., 32(18), L18801, doi:10.1029/2005GL023614, 2005.

Prenni, A. J., DeMott, P. J., Twohey, C., et al.: Examinations of ice formation processes in Florida cumuli using ice nuclei measurements of anvil ice crystal particle residues, J. Geophys. Res.Atmos., 112(D10), D10221, doi:10.1029/2006JD007549, 2007.

Pruppacher, H. R. and Klett, J. D.: Microphysics of Clouds and Precipitation, Kluwer Academic Publishers, Dordrecht, Boston, London, 954 pp., 1997

Seeley, L. H. and Seidler, G. T.: Preactivation in the nucleation of ice by Langmuir films of aliphatic alcohols, J. Chem. Phys., 114(23), 10 464-10470, 2001a.

Seeley, L. H. and Seidler, G. T.: Two-dimensional nucleation of ice from supercooled water, Phys. Rev. Lett., 87(5), 055702, doi:10.1103/PhysRevLett.87.055702, 2001b.

Schnell, R. C. and Vali, G.: Ice nuclei from decomposing vegetation, Nature, 236(5343), 163-164, 1972.

Shaw, R. A., Durant, A. J., and Mi, Y.: Heterogeneous surface crystallization observed in undercooled water, J. Phys. Chem. B, 109, 9865-9868, 2005.

Vali, G.: Quantitative evaluation of experimental results on the heterogeneous freezing nucleation of supercooled liquids, J. Atmos. Sci., 28, 402-409, 1971.

Vali, G.: Freezing rate due to heterogeneous nucleation, J. Atmos. Sci., 51(13), 1843-1856, 1994.

Vali, G. and Stansbury, E. J.: Time dependent characteristics of the heterogeneous nucleation of ice, Can. J. Phys., 44, 477-502, 1966.

Volmer, M.: Kinetic der Phasenbildung, Steinkopf, Leipzig, Leipzig, 325 pp., 1939.

Vonnegut, B. and Baldwin, M.: Repeated nucleation of a supercooled water sample that contains silver iodide particles, J. Appl. Meteor., 23(3), 486-490, 1984.

Zobrist, B., Koop, T., Luo, B. P., Marcolli, C., and Peter, T.: Heterogeneous ice nucleation rate coefficient of water droplets coated by a nonadecanol monolayer, J Phys. Chem. C, 111(5), 21492155, 2007. 